\title{
Motion-corrected whole-heart PET-MR for the simultaneous visualisation of coronary artery integrity and myocardial viability: an initial clinical validation
}

\author{
Camila Munoz ${ }^{1}$ (D) Karl P. Kunze $^{2} \cdot$ Radhouene Neji $^{1,3} \cdot$ Teresa Vitadello $^{2} \cdot$ Christoph Rischpler $^{2} \cdot$ René M. Botnar $^{1,4}$. \\ Stephan G. Nekolla ${ }^{2,5} \cdot$ Claudia Prieto ${ }^{1,4}$
}

Received: 14 February 2018 / Accepted: 2 May 2018 / Published online: 12 May 2018

(C) The Author(s) 2018

\begin{abstract}
Purpose Cardiac PET-MR has shown potential for the comprehensive assessment of coronary heart disease. However, image degradation due to physiological motion remains a challenge that could hinder the adoption of this technology in clinical practice. The purpose of this study was to validate a recently proposed respiratory motion-corrected PET-MR framework for the simultaneous visualisation of myocardial viability $\left({ }^{18} \mathrm{~F}-\mathrm{FDG} \mathrm{PET}\right)$ and coronary artery anatomy (coronary MR angiography, CMRA) in patients with chronic total occlusion (CTO).

Methods A cohort of 14 patients was scanned with the proposed PET-CMRA framework. PET and CMRA images were reconstructed with and without the proposed motion correction approach for comparison purposes. Metrics of image quality including visible vessel length and sharpness were obtained for CMRA for both the right and left anterior descending coronary arteries (RCA, LAD), and relative increase in ${ }^{18}$ F-FDG PET signal after motion correction for standard 17-segment polar maps was computed. Resulting coronary anatomy by CMRA and myocardial integrity by PET were visually compared against X-ray angiography and conventional Late Gadolinium Enhancement (LGE) MRI, respectively.

Results Motion correction increased CMRA visible vessel length by $49.9 \%$ and $32.6 \%$ (RCA, LAD) and vessel sharpness by $12.3 \%$ and $18.9 \%$ (RCA, LAD) on average compared to uncorrected images. Coronary lumen delineation on motion-corrected CMRA images was in good agreement with X-ray angiography findings. For PET, motion correction resulted in an average 8\% increase in ${ }^{18}$ F-FDG signal in the inferior and inferolateral segments of the myocardial wall. An improved delineation of myocardial viability defects and reduced noise in the ${ }^{18} \mathrm{~F}$-FDG PET images was observed, improving correspondence to subendocardial LGE-MRI findings compared to uncorrected images.

Conclusion The feasibility of the PET-CMRA framework for simultaneous cardiac PET-MR imaging in a short and predictable scan time ( $\sim 11 \mathrm{~min}$ ) has been demonstrated in 14 patients with CTO. Motion correction increased visible length and sharpness of the coronary arteries by CMRA, and improved delineation of the myocardium by ${ }^{18} \mathrm{~F}-\mathrm{FDG}$ PET, resulting in good agreement with X-ray angiography and LGE-MRI.
\end{abstract}

Keywords Cardiac PET-MR · Motion correction · Coronary artery disease · Coronary MR angiography

Stephan G. Nekolla and Claudia Prieto contributed equally to this work.

Camila Munoz

camila.munoz@kcl.ac.uk

1 School of Biomedical Engineering and Imaging Sciences, King's College London, 4th Floor, Lambeth Wing, St Thomas' Hospital, London SE1 7EH, UK

2 Nuklearmedizinische Klinik und Poliklinik, Technische Universität München, Munich, Germany

3 MR Research Collaborations, Siemens Healthcare, Frimley, UK

4 Escuela de Ingenieria, Pontificia Universidad Catolica de Chile, Santiago, Chile

5 DZHK (Deutsches Zentrum für Herz-Kreislauf-Forschung e.V.) partner site Munich Heart Alliance, Munich, Germany 


\section{Introduction}

Simultaneous PET-MR imaging has shown promising results for the comprehensive assessment of several cardiac conditions in initial clinical studies [1-5]. Dweck et al. [6] observed increased accuracy for the diagnosis of active cardiac sarcoidosis by combining ${ }^{18} \mathrm{~F}$-fluorodeoxyglucose $\left({ }^{18} \mathrm{~F}\right.$-FDG) PET and cardiovascular MR (CMR). Nensa et al. [7] showed promising results of increased sensitivity for the detection of borderline myocarditis using hybrid ${ }^{18} \mathrm{~F}-\mathrm{FDG}$ PET and CMR scans. A significant PET-MR intermodality agreement was observed in acute myocardial infarction [8], and more recently $\mathrm{CMR}$ in combination with ${ }^{18} \mathrm{~F}$-FDG and ${ }^{18} \mathrm{~F}$-sodium fluoride $\left({ }^{18} \mathrm{~F}-\mathrm{NaF}\right)$ was successfully used for imaging of inflammation and calcification in the coronary arteries [9].

Most of the currently used cardiac PET-MR imaging workflows consist of the acquisition of several MR images with differing contrasts, while simultaneously acquiring list-mode PET data throughout the whole or most of the scanning session. Cardiac MR images acquired in these protocols typically include a stack of cine images, Late Gadolinium Enhancement (LGE), coronary MR angiography (CMRA) and $T_{1}$ and $T_{2}$ mapping, leading to scanning sessions that range between 30 and $90 \mathrm{~min}$ in duration $[6,8,9]$. During this prolonged examination time, physiological motion occurs due to breathing and cardiac motion (contraction and relaxation), thus requiring motion compensation techniques to minimise ghosting, blurring and associated image artefacts in both modalities.

Cardiac motion compensation is typically achieved in MR by synchronising the acquisition with an external electrocardiogram (ECG) device and acquiring data only during the quiescent periods of the cardiac cycle, e.g. mid-diastole. Respiratory motion is conventionally addressed in a similar fashion, by acquiring and accepting data only during a specific respiratory phase of minimal motion, typically end-expiration. Surrogate signals that have a strong correlation with the respiratory motion of the heart are used for synchronisation, either from external devices (such as respiratory bellows) or imagederived signals (such as monitoring the position of the lungliver interface, so-called 1D image navigators) [10]. These approaches, however, lead to long and unpredictable scan times due to breathing pattern variations and drifting. Similar approaches have been proposed for cardiac PET imaging [11], so that the acquired list-mode data are dual-gated into a number of cardiac and respiratory phases, and only data acquired during end-expiration and mid-diastole are used for image reconstruction. However, the significant reduction in data available in each gate produces images with increased noise. Therefore, in practice, most of the clinical studies still aggregate all the acquired data into a single static time frame blurred by both respiratory and cardiac motion $[6,8]$.

Simultaneous PET-MR technology has shown potential for addressing the motion problem in PET by incorporating motion information estimated from simultaneously acquired MR images into PET image reconstruction. In order to obtain motion information, a set of MR images is acquired at different cardiac and respiratory positions, and motion is estimated by non-rigid registration of the images for each frame, typically using the end-diastolic and end-expiration frame as reference [12]. The simultaneously acquired PET data is then sorted into corresponding frames, and MR-based motion fields are incorporated into the PET image reconstruction $[13,14]$ to reduce motion induced blurring and artefacts, improving PET image quality and quantification [12].

In most of the current approaches for MR-based motion correction of cardiac PET data, the MR images acquired simultaneously with PET are designed for motion estimation purposes only [15-17]. The lack of appropriate tissue contrast and/or sufficient spatial resolution of these MR images might prevent their use for diagnostic purposes. This may lead to prolonged examination times, since diagnostic MR images are to be acquired after the simultaneous PET-MR examination.

We have recently proposed a different approach for the acquisition of motion-corrected cardiac PET-MR images [18]. With this approach motion estimated from MR images is used to correct both ${ }^{18} \mathrm{~F}$-FDG PET and CMRA datasets, enabling visualisation of myocardial viability and coronary anatomy in a single efficient examination. This PET-CMRA motion correction framework is based on 2D image-based navigation $[19,20]$, thus allowing for $100 \%$ scan efficiency (no respiratory gating window with concomitant data rejection) and predictable scan time. The feasibility of this PETCMRA framework was shown in a small cohort $(N=5)$ of oncology patients without known or suspected coronary artery disease (CAD) [18], however its feasibility in patients with cardiovascular disease has not been investigated yet. Here we present an initial clinical validation of this method for the simultaneous visualisation of myocardial integrity by ${ }^{18} \mathrm{~F}$ FDG PET and coronary lumen integrity by CMRA. The study was performed in a cohort of patients with known CAD, in particular, patients with chronic total occlusion of at least one of the coronary arteries.

\section{Materials and methods}

\section{PET-MR image acquisition}

The whole-heart PET-CMRA acquisition consists of a freebreathing ECG-triggered CMRA sequence simultaneously acquired with list-mode cardiac PET data (Fig. 1a) as proposed in [18]. CMRA data is acquired using a 3D spoiled gradient echo sequence following a fully sampled golden-step Cartesian trajectory with spiral profile ordering [21], so that one spiral interleaf is acquired at each heartbeat. A low- 
(a)

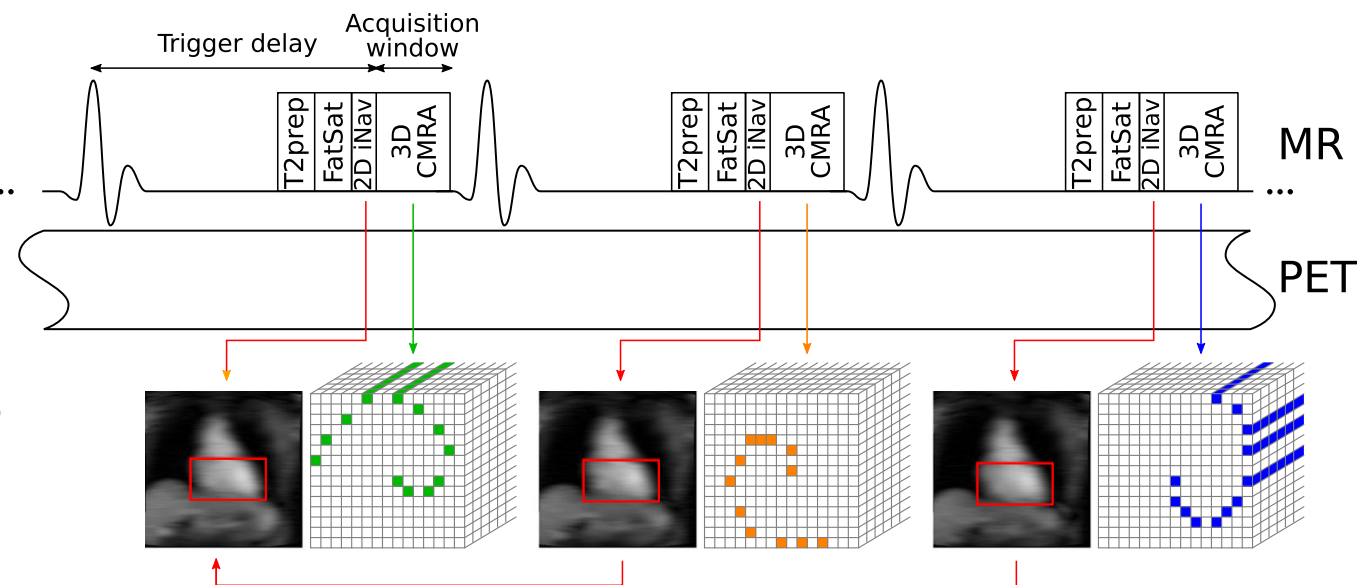

(b)

Rigid registration

(c)

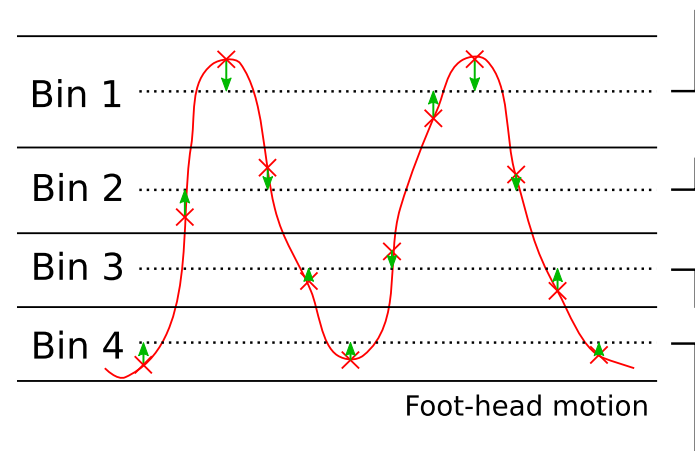

(e)

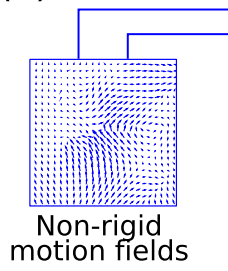

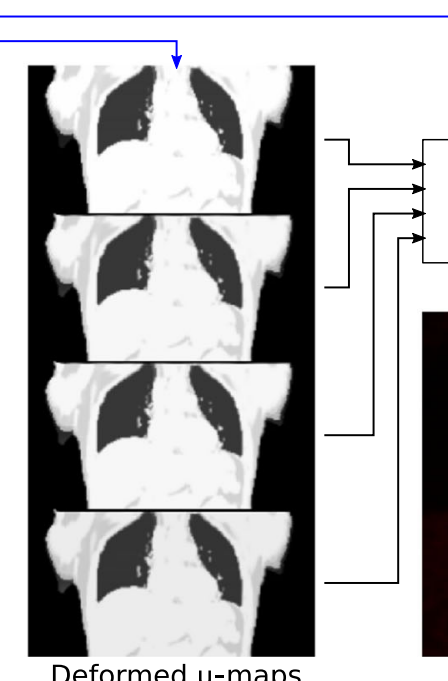

Deformed $\mu$-maps

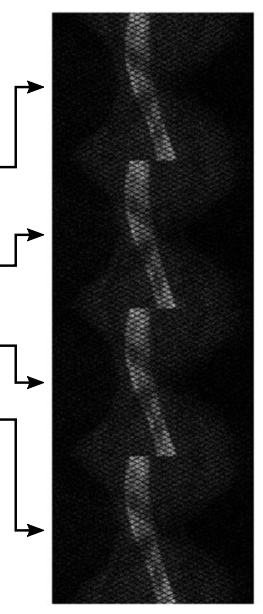

Binned PET data

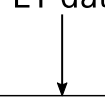

Motion corrected PET reconstruction

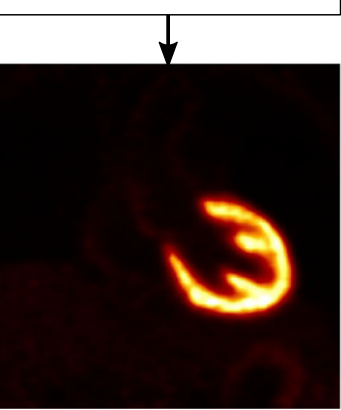

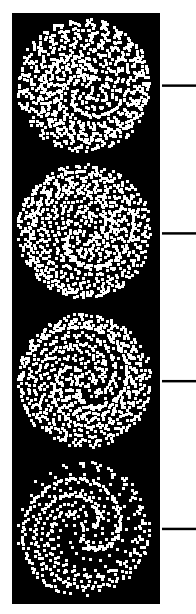

Binned MR data

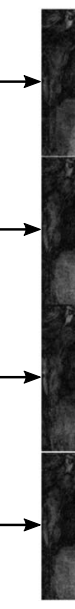

MR bins

data

(d)
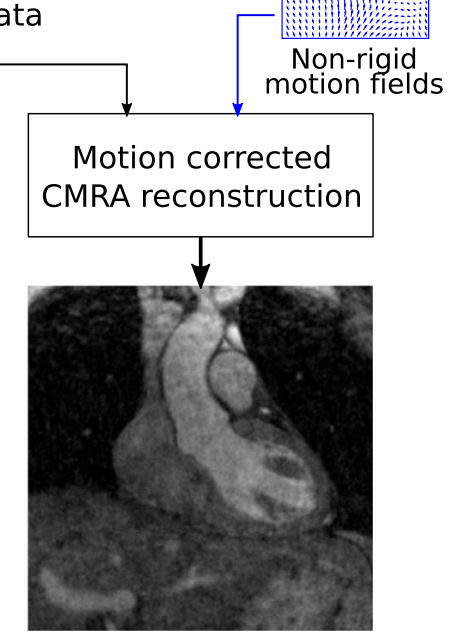

Fig. 1 PET-CMRA acquisition and reconstruction scheme. a PETCMRA acquisition sequence, an image-navigator (2D iNAV) is acquired at each heartbeat before the CMRA acquisition for estimating respiratory motion. T2prep and FatSat preparation pulses are performed to improve contrast between blood and surrounding tissue. b Translational motion in FH and RL directions is estimated from the 2D iNAVs by rigid registration of a template covering the apex of the heart (in red). $\mathbf{c}$ FH motion is used to bin the PET and CMRA data in a number of respiratory windows. FH and RL motion is used to correct the CMRA data to the centre of each bin (green arrows). d Non-rigid deformation fields are estimated from MR images reconstructed at each respiratory position and e used to move the attenuation maps to each respiratory position, and in motion compensated PET and CMRA image reconstruction 
resolution coronal 2D image navigator (iNAV) [19] for respiratory motion estimation is acquired at the beginning of each interleaf by spatially encoding low flip angle $\mathrm{k}$-space lines. Fat saturation (Fat Sat) and T2-preparation (T2-prep) [22] pulses are performed immediately prior to data acquisition to improve the contrast between arterial blood and the surrounding myocardium and epicardial fat.

As part of the cardiac PET-CMRA acquisition protocol, a standard Dixon-based attenuation map ( $\mu$-map) is acquired in breath-hold at end-expiration for MR-based attenuation correction of the PET data [23], with missing tissue due to the limited field of view of the MR (as compared to PET) estimated using the MLAA (Maximum Likelihood reconstruction of Attenuation and Activity) approach [24]. Additionally, a conventional $2 \mathrm{D}$ cine image is acquired for defining the trigger delay and length of the acquisition window of the 3D CMRA.

\section{PET-MR image reconstruction}

Motion compensated image reconstruction is performed in four steps (Fig. 1b-e). In the first step, foot-head (FH) and right-left (RL) translational respiratory motion is estimated from the 2D iNAVs by using rigid image registration of a template covering the apex of the heart (Fig. 1b). This allows measuring directly the respiratory-induced translational motion of the heart in a beat-to-beat fashion. In the second step, FH motion is used to bin the acquired PET and CMRA data in a number of respiratory windows ranging from end-expiration to end-inspiration, each containing the same amount of data (Fig. 1c). Outlier rejection is performed in this step, by excluding CMRA data acquired in deep breaths [18]. CMRA data acquired within each respiratory window is then corrected to the centre of the bin by applying a phase shift in k-space according to the estimated FH and RL motion. In a third step, 3D MR images are reconstructed at each respiratory position using iterative SENSE with a soft-binning approach $[18,20]$, and bin-to-bin respiratory deformation fields are estimated by non-rigid image registration, using the end-expiration bin as reference (Fig. 1d). Finally, the non-rigid deformation fields are used in a generalised matrix description formulation for motion-compensated CMRA reconstruction [25]. Moreover, the non-rigid motion fields are also used to move the attenuation maps to each respiratory position (Fig. 1e) and perform a motion-compensated PET reconstruction. Therefore, at the end of the reconstruction process, co-registered respiratory motion-corrected CMRA and cardiac PET images are obtained.

\section{Experiments}

Fourteen patients with symptomatic CAD (angina or angina equivalent, excluding acute STEMI patients), chronic total occlusion (CTO) of a relevant coronary artery (segment 1, 2, $6,7,11$ or 13 , diameter $>2.5 \mathrm{~mm}$ ), and echocardiographic or angiographic evidence of a wall motion abnormality in the corresponding area were recruited for this study between October 12, 2016 and June 26, 2017 (Fig. 2). In order to improve risk stratification before elective Percutaneous Coronary Intervention (PCI) of the $\mathrm{CTO}$, all patients underwent a hybrid ${ }^{18}$ F-FDG PET-MR examination in a Biograph mMR scanner (Siemens Healthcare, Erlangen, Germany). Relevant patient characteristics include: age 66.1 \pm 9.5 years, 9 males, 5 females, LVEF $49 \pm 12$, and previous PCI stenting of at least one of the coronary arteries. Written informed consent with respect to participation was obtained from all subjects; the study was performed in concordance with the Declaration of Helsinki and approved by the institutional ethics committee.

The clinical PET-MR examination protocol included a scan of 40 to $50 \mathrm{~min}$ with a list-mode PET acquisition for the assessment of myocardial viability under insulin-clamped conditions $60 \mathrm{~min}$ after injection of $326 \pm 29 \mathrm{MBq}$ of ${ }^{18} \mathrm{~F}$ FDG, and a multi-slice 2D-Phase Sensitive Inversion Recovery (PSIR) LGE acquisition (1.4-2.2 mm in-plane resolution, $8 \mathrm{~mm}$ slice thickness). During the $10-15$ min waiting time required for optimal contrast in LGE images, an acquisition with a prototype implementation of the proposed PETCMRA sequence was performed.

For the CMRA acquisition, relevant imaging parameters included: coronal orientation, resolution $=1 \times 1 \times 2 \mathrm{~mm}^{3}$ (interpolated to $1 \mathrm{~mm}^{3}$ isotropic resolution during image reconstruction), field of view $=304 \times 304 \times 88-112 \mathrm{~mm}^{3}$ covering the whole heart, TR/TE $=3.72 / 1.70 \mathrm{~ms}$, and flip angle $=15^{\circ}$. A subject-specific trigger delay was defined targeting the middiastolic quiescent period of the cardiac cycle, and an

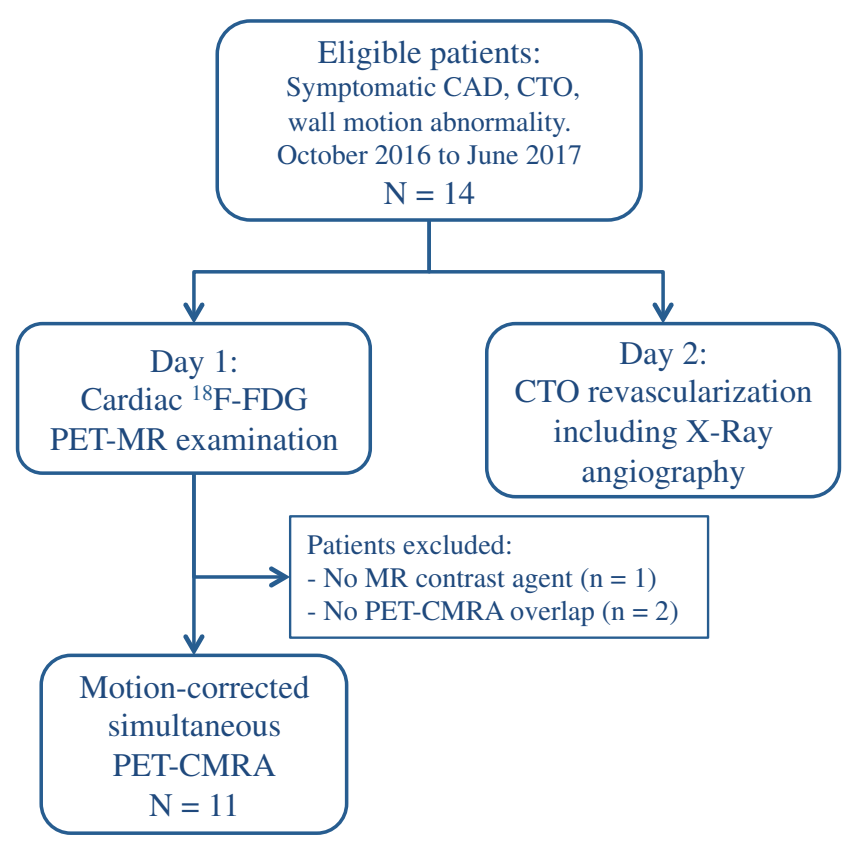

Fig. 2 Flow chart of patients included in the study 
acquisition window of 89 to $119 \mathrm{~ms}$ (corresponding to 24 to 32 lines per spiral interleaf) was selected depending on the length of the mid-diastolic period. The 2D iNAVs were acquired using the following parameters: same field of view as the CMRA acquisition, flip-angle $=3^{\circ}, 14$ acquired lines with a centric-in Cartesian trajectory, corresponding to a $1 \times$ $21.7 \mathrm{~mm}^{2}$ acquired resolution, interpolated to $1 \times 1 \mathrm{~mm}^{2}$ before FH and RL motion estimation. The vendor-provided Fat Sat prepulse was used, and an adiabatic T2-prep (50 ms duration) was implemented to improve tissue contrast. $\mu$-maps were acquired for each patient during a $19 \mathrm{~s}$ breath-hold at end-expiration using the vendor's standard Dixon protocol (acquisition parameters: coronal orientation, resolution $=$ $2.6 \times 2.6 \times 3.1 \mathrm{~mm}^{3}$, field of view $=328 \times 500 \times 399 \mathrm{~mm}^{3}$, $\mathrm{TR} / \mathrm{TE} 1 / \mathrm{TE} 2=3.60 / 1.23 / 2.46 \mathrm{~ms})$. All patients underwent interventional X-ray angiography the day after the PET-MR examination, for elective $\mathrm{CTO}$ revascularization.

CMRA and PET datasets were reconstructed with the described motion correction scheme (MC) and without motion correction (NMC) for comparison purposes. For each patient, the fraction of list-mode PET data acquired simultaneously with the CMRA sequence was selected for PET image reconstruction. Due to variations in planning time, the overlap between CMRA and list-mode PET acquisition varied between patients, with an average overlap of $80.3 \pm 20.9 \%$ of the duration of the CMRA acquisition.

MR image reconstruction was performed offline in MATLAB (Mathworks, Natick, Massachusetts, USA) using custom developed software. PET image reconstruction was performed offline using reconstruct-transform-average [13] motion correction. For this, the standard $\mu$-map acquired at end-expiration was moved to each respiratory position in MATLAB using the deformation fields estimated from MR images. Each respiratory bin was independently reconstructed offline with e7 Tools (Siemens Healthcare, Knoxville, USA) using the OSEM algorithm, with three iterations and 21 subsets, point spread function modelling, voxel size $=2.03 \times$ $2.08 \times 2.08 \mathrm{~mm}^{3}$, and matrix size $=127 \times 344 \times 344$. Finally, images reconstructed at each respiratory position were combined in MATLAB to produce a motion corrected PET image.

\section{Image analysis}

Reconstructed CMRA images were reformatted to simultaneously visualise the left anterior descending (LAD) artery and right coronary artery (RCA) using dedicated software [26]. Metrics of visible vessel length and sharpness were obtained for vessels without stents in the proximal artery. Vessel sharpness values were normalised to the signal intensity of the centre line of each vessel, so that $100 \%$ sharpness refers to a maximum signal intensity change at the vessel edge. Additionally, motion corrected CMRA images were reformatted following the anatomy observed in the X-ray angiography in order to compare both visually.

Reconstructed ${ }^{18}$ F-FDG PET images were analysed by automatically applying the 17 -segment model according to the American Heart Association [27] to the left ventricular myocardium using dedicated software (MunichHeart [28]). The relative increase in ${ }^{18} \mathrm{~F}$-FDG signal of motion corrected images over uncorrected images was computed for each of the 17 segments for each patient. Additionally, NMC and MC PET images were reoriented in short axis to visually compare them with LGE images.

\section{Results}

Scans were successfully completed in all subjects. The average acquisition time for the proposed PET-CMRA framework was $11.2 \pm 2.4 \mathrm{~min}$. Due to differences in planning time, in two of the patients there was no overlap between the PET and the CMRA acquisition, so that motion correction of the PET data could not be performed. Additionally, one of the patients was not able to receive the gadoliniumbased contrast agent. These three patients were excluded from the following analysis. For the rest of the patients $(N=11)$, the average overlap between PET and CMRA acquisition was $8.8 \pm 1.2 \mathrm{~min}$, corresponding to list-mode PET data being acquired during $80.3 \pm 20.9 \%$ of the duration of the CMRA acquisition on average.

Reformatted images showing non-stented RCA and LAD for three of the patients are shown in Fig. 3. Improvements in the delineation of the RCA and LAD were observed in the CMRA images after applying MC, allowing for depiction of non-stented vessels, even in cases where severe respiratory motion prevented the visualisation of both the left and right proximal coronary arteries in the NMC image, as observed for Patient 1 . Figure 4 shows example coronal slices of the ${ }^{18} \mathrm{~F}$ FDG PET images reconstructed for three representative patients. MC increased the sharpness of the left ventricle myocardium and improved visualisation of small features such as the papillary muscles (blue arrows) and delineation of viability defects (red arrow) compared to NMC.

A visual comparison between the reformatted MC CMRA and the corresponding invasive X-ray angiogram is shown in Fig. 5 for two patients. Adequate spatial resolution and contrast in CMRA images allowed for a depiction of the proximal arteries comparable to the X-ray angiogram for both cases. In Patient 6, a stenosis observed in the mid segment of the RCA in CMRA was confirmed in the angiogram (Fig. 5, red arrows), while in Patient 7 an aneurysm in the proximal RCA was seen in both modalities (Fig. 5, green arrows).

Figure 6 shows a short axis view of the ${ }^{18} \mathrm{~F}$-FDG PET both for $\mathrm{NMC}$ and $\mathrm{MC}$ reconstructions and corresponding slice of the 2D LGE scan for two patients. It can be observed that MC 

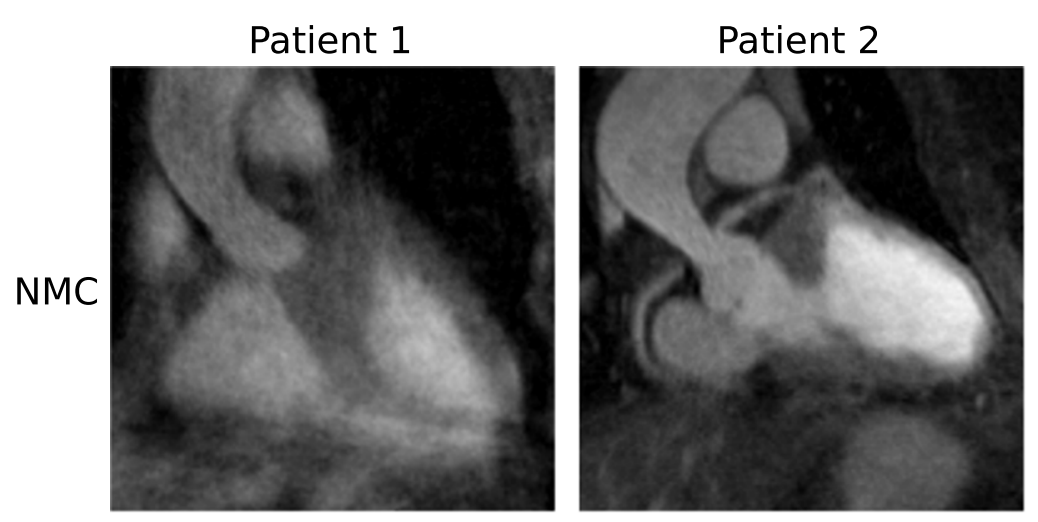

Patient 3
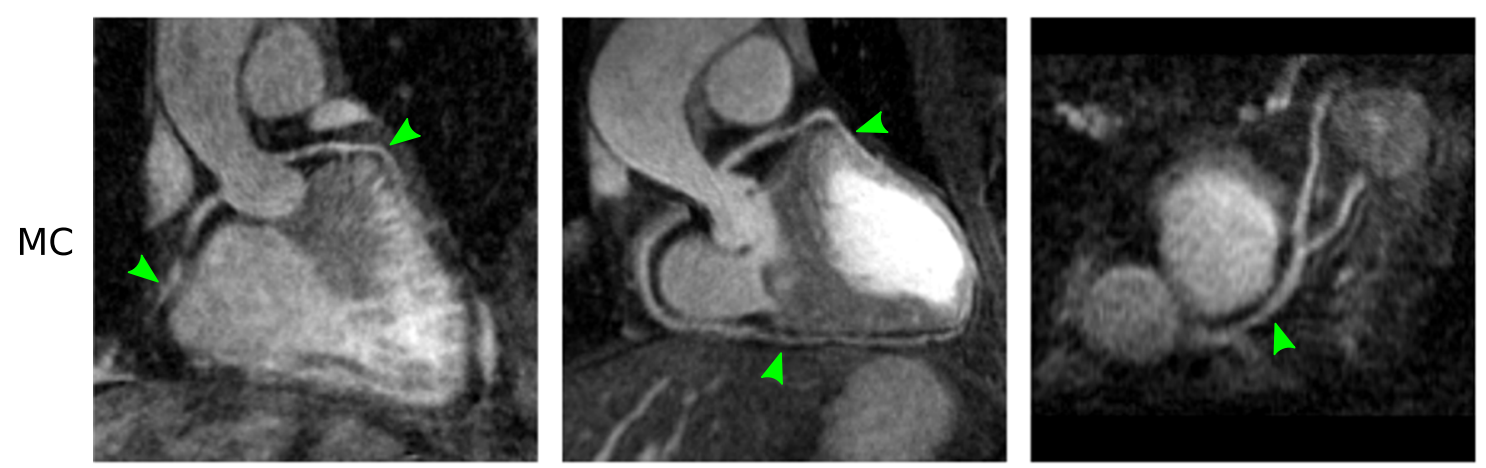

Fig. 3 Reformatted images for three representative patients (columns) showing uncorrected (NMC) and motion-corrected (MC) CMRA. Improvements in the visualisation of the vessels are observed when applying MC (green arrows) for all cases, particularly in the distal segments of the arteries
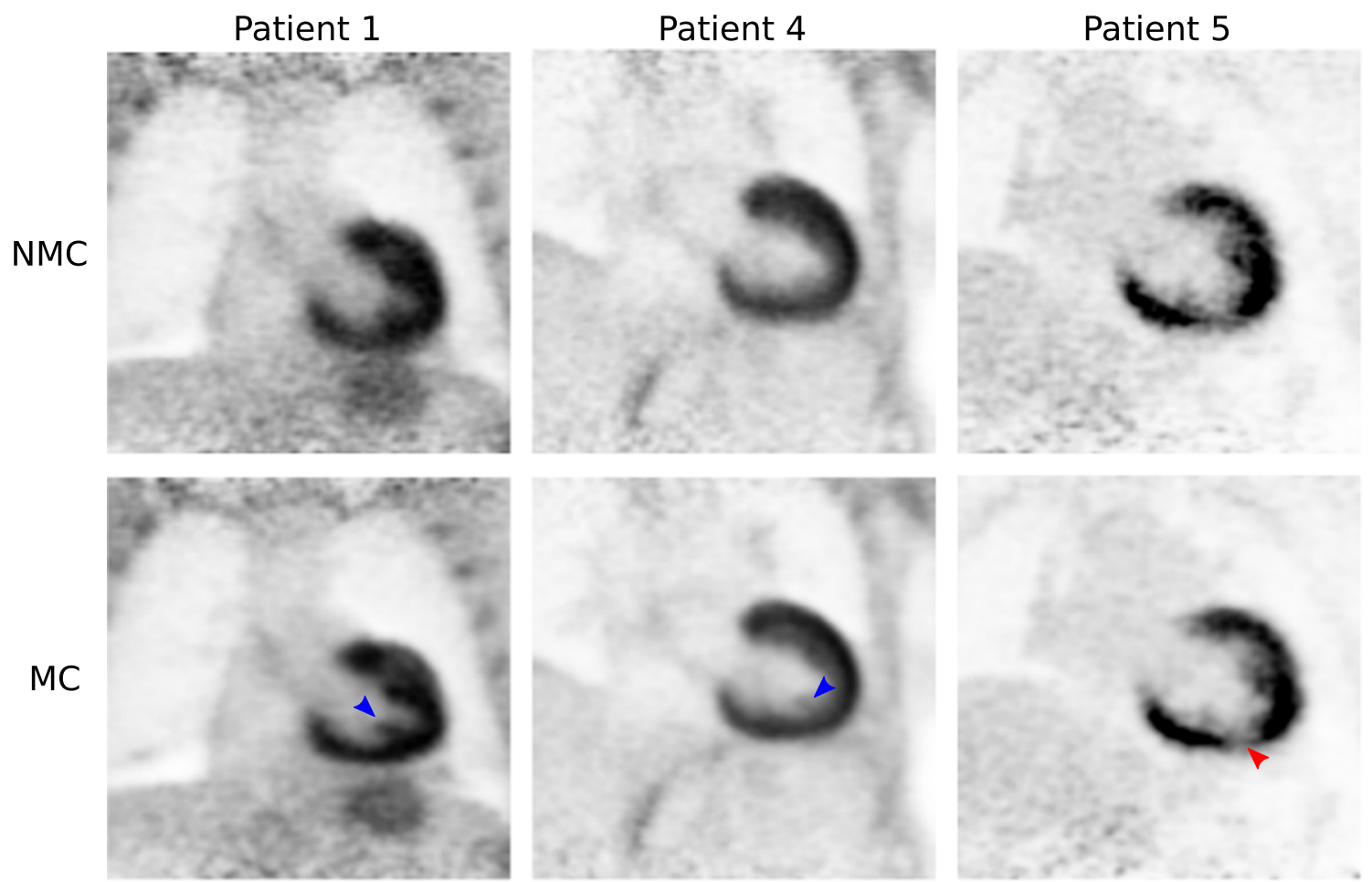

Fig. 4 Coronal slice for three representative patients (columns) showing uncorrected (NMC) and motion-corrected (MC) viability ${ }^{18} \mathrm{~F}-\mathrm{FDG}$ PET images. Improvements in image quality can be observed when applying MC, particularly in small structures (blue and red arrows), compared to NMC 
Fig. 5 Reformatted CMRA and corresponding X-ray angiogram for two representative patients. Red arrows: stenosis in the mid segment of the right coronary artery (RCA); green arrows: aneurysm in the proximal segment of the RCA
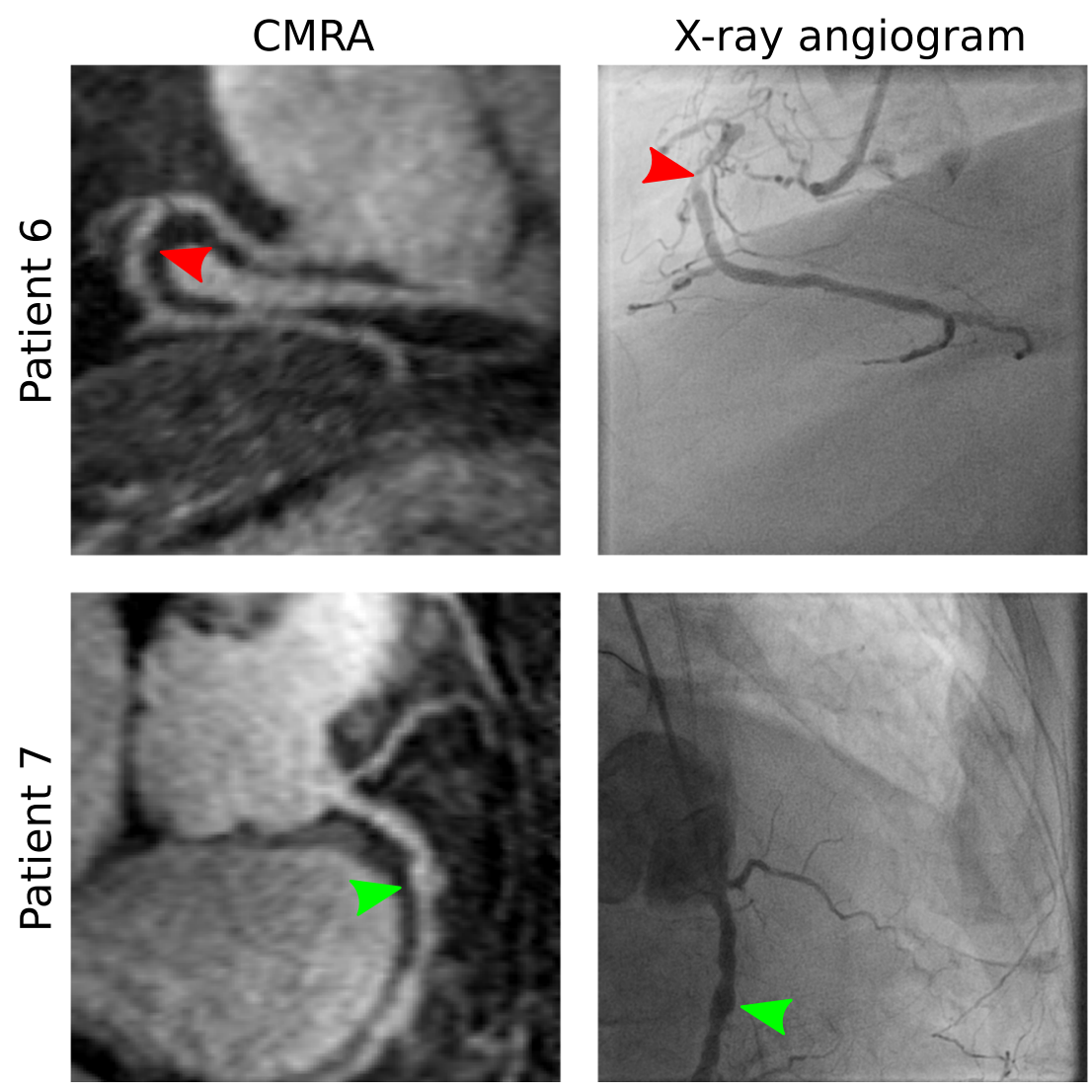

PET MC
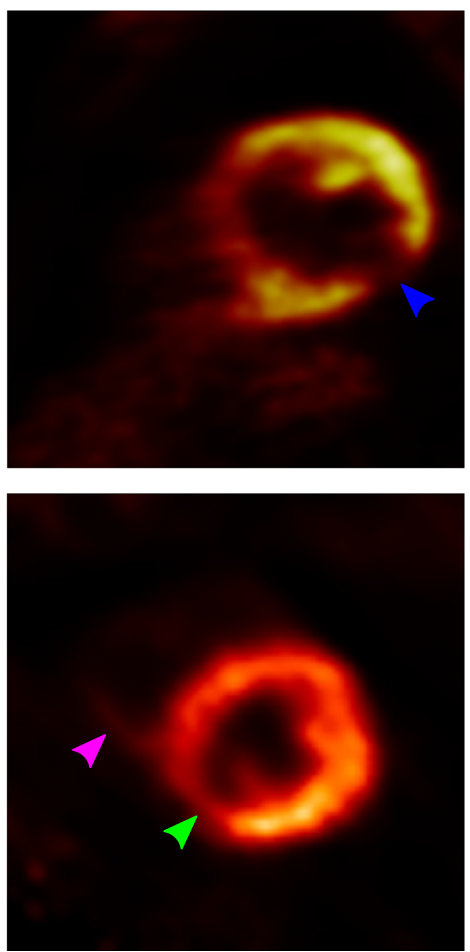

LGE
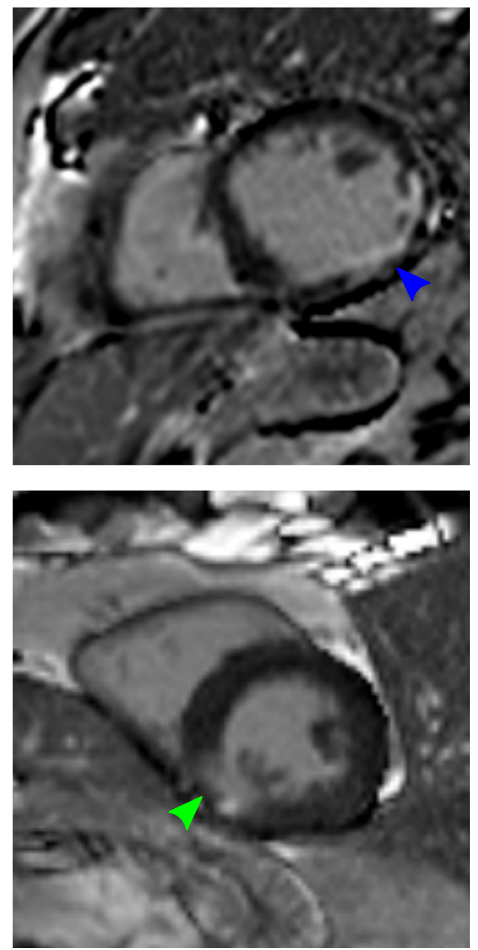

Fig. 6 Short axis view for two representative patients (rows) showing uncorrected (NMC) and motion-corrected (MC) viability ${ }^{18} \mathrm{~F}$-FDG PET images, and corresponding 2D LGE scans. MC improves the correspondence of the PET images to the anatomy as observed in the LGE images, particularly in the delineation of viability defects (green and blue arrows) and right ventricle myocardium (pink arrow) 
PET images have an improved correspondence to the anatomy as observed in the LGE images and reduced noise compared to NMC images. In particular, improvements in delineation of viability defects are apparent: for Patient 6 , the transmural viability defect observed in the infero-lateral wall was better depicted after motion correction (Fig. 6, blue arrows), while in Patient 8 , motion correction allowed for the identification of viable myocardium in a defect that appeared misleadingly as transmural in the NMC image (Fig. 6, green arrows). MC also enabled the depiction of thinner structures, such as right ventricle myocardium (Fig. 6, pink arrow).

Image quality metrics for the NMC and MC CMRA images are shown in Fig. 7. Tracking of the vessels was possible in 14 out of 15 non-stented vessels (8 RCA, 6 LAD). In one of the patients, significant cardiac motion prevented the visualisation of the coronary arteries. For the rest of the vessels, increased visible length of the vessels were observed after $\mathrm{MC}$ for both the RCA ( $+49.9 \%$ on average) and the LAD $(+32.6 \%$ on average) (Fig. 7a, c). Similarly, vessel sharpness increased by $12.3 \%$ and $18.9 \%$ on average for the proximal RCA and LAD respectively when using MC (Fig. 7b, d).

Polar maps showing the relative increase in ${ }^{18} \mathrm{~F}-\mathrm{FDG}$ PET signal after motion correction in the left ventricular myocardium are displayed in Fig. 8 for two patients with distinct respiratory patterns (Fig. 8a-b) and on average across the cohort (Fig. 8c). For the patient with significant respiratory motion (Fig. 8a), the average signal increased across all segments was $33 \%$, with a maximum in the anterior wall. For the patient with moderate respiratory motion (Fig. 8b), an average of $3 \%$ signal increase was observed across all segments. When considering the cohort averages for each myocardial segment (Fig. 8c), signal increased most towards the inferior and inferolateral regions, i.e. by an average of $8 \%$ across the six corresponding sectors of the myocardium.

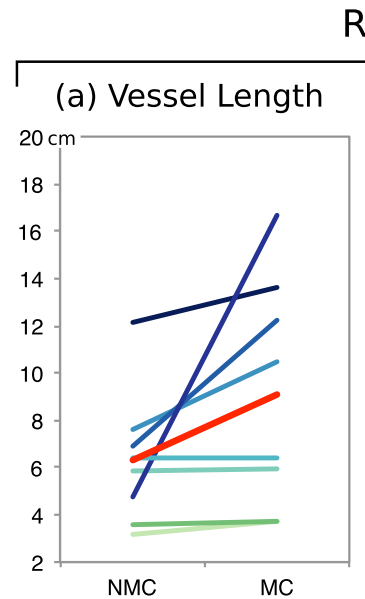

RCA
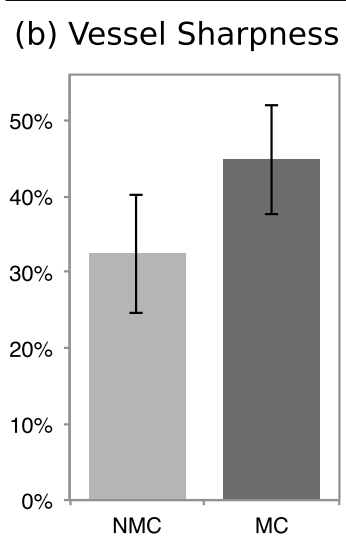

Fig. 7 Image quality analysis for uncorrected (NMC) and motioncorrected (MC) CMRA images in 11 patients, including: visible vessel length for (a) RCA and (c) LAD, for each of the patients (green/blue lines) and average (red line); and vessel sharpness for (b) RCA and (d)
Figure 9 shows an example fused PET-CMRA dataset before and after motion correction. The framework produces coregistered diagnostic PET and CMRA images, improving the correspondence between modalities compared to uncorrected images.

\section{Discussion}

In this study we have tested a novel approach for the simultaneous visualisation of coronary anatomy by CMRA and myocardial viability by ${ }^{18} \mathrm{~F}$-FDG PET in patients with coronary artery disease. The framework incorporates $2 \mathrm{D} \mathrm{im}-$ age-navigation, and uses motion estimated from MR to correct both the PET and CMRA datasets, producing respiratory motion-corrected co-registered images. In contrast to recently proposed MR-based motion correction approaches for cardiac PET data that utilise MR images mainly for improving PET image quality [15-17], our approach produces diagnostic images in both modalities, potentially reducing total examination time. Furthermore, the proposed PETCMRA acquisition and reconstruction scheme has a short and predictable scan time of approximately $11 \mathrm{~min}$, which makes it suitable for clinical practice. Indeed, for this study the proposed PET-CMRA acquisition was included into a standard clinical PET-MR protocol during the waiting time required for conventional LGE imaging.

The motion correction framework for CMRA data has been previously shown and validated for healthy subjects $[18,20]$, but to the best of the authors' knowledge, this is the first study that shows the feasibility of applying such framework to patients with cardiovascular disease. For the cohort of patients in this study, improvement in image quality after motion correction is apparent, allowing for the visualisation

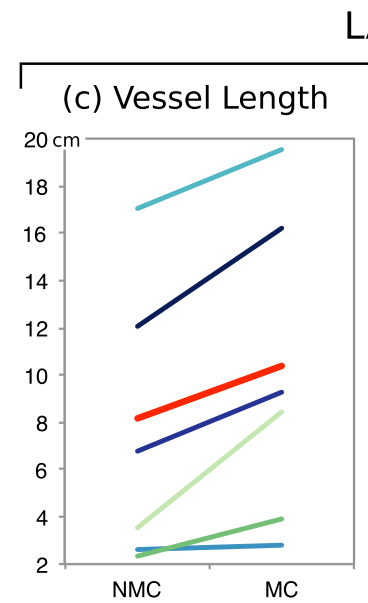

AD

LAD. Visible vessel length and sharpness improve after motion correction for all cases. Image analysis was performed in vessels without stents in the proximal segment 


\section{Significant respiratory motion}

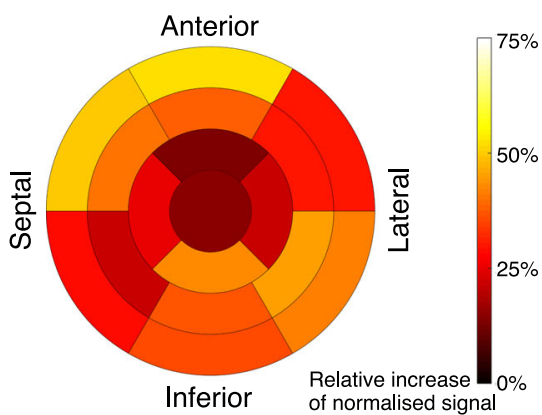

Respiratory belt signal

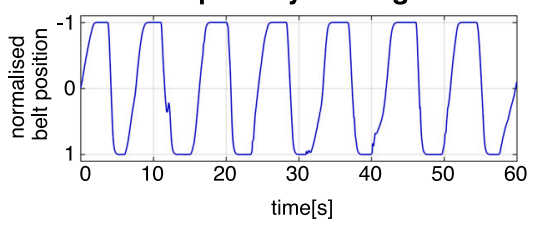

Modest respiratory motion

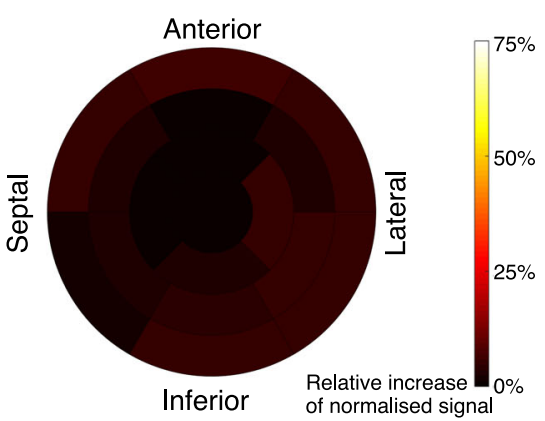

Respiratory belt signal

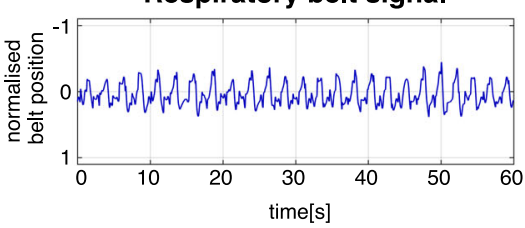

Cohort average

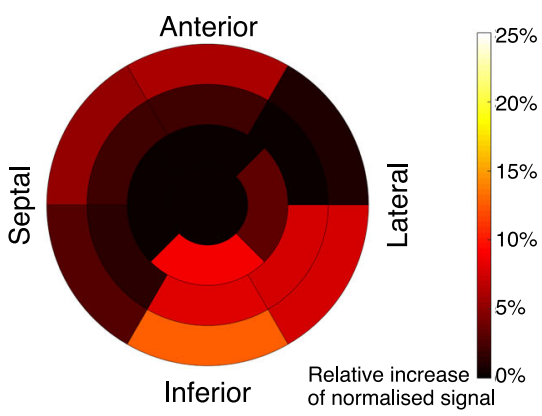

Fig. 8 17-segment polar maps of the relative increase in ${ }^{18}$ F-FDG PET signal after motion correction for the left ventricular myocardium. Representative patients with (a) high amplitude respiratory motion and (b) moderate respiratory motion, and (c) the average across the cohort

of 14 out of 15 non-stented vessels and increasing visible length and sharpness of the coronary arteries. Furthermore, visual comparison showed good agreement between the motion-corrected CMRA and gold standard invasive Xray angiograms, including visualisation of stenosis and aneurysm in the proximal segment of the arteries (Fig. 5).

For the ${ }^{18}$ F-FDG myocardial viability PET images, the proposed motion correction approach improved the depiction of small structures such as the right ventricle myocardium and papillary muscles, and enhanced the visualisation of transmural and non-transmural viability defects in the left ventricle myocardium. In general, motion correction produced an increased PET signal in each of the myocardial segments, with an average of $8 \%$ relative increase for the inferolateral wall. When analysing the relative signal increase for each dataset, it was observed that improvements were strongly related to the respiratory pattern of each subject: modest increase in signal was obtained in patients with shallow breathing, while increases of up to $33 \%$ on average were observed in patients with significant respiratory motion. Such increases come both from a reduction in the blurring and
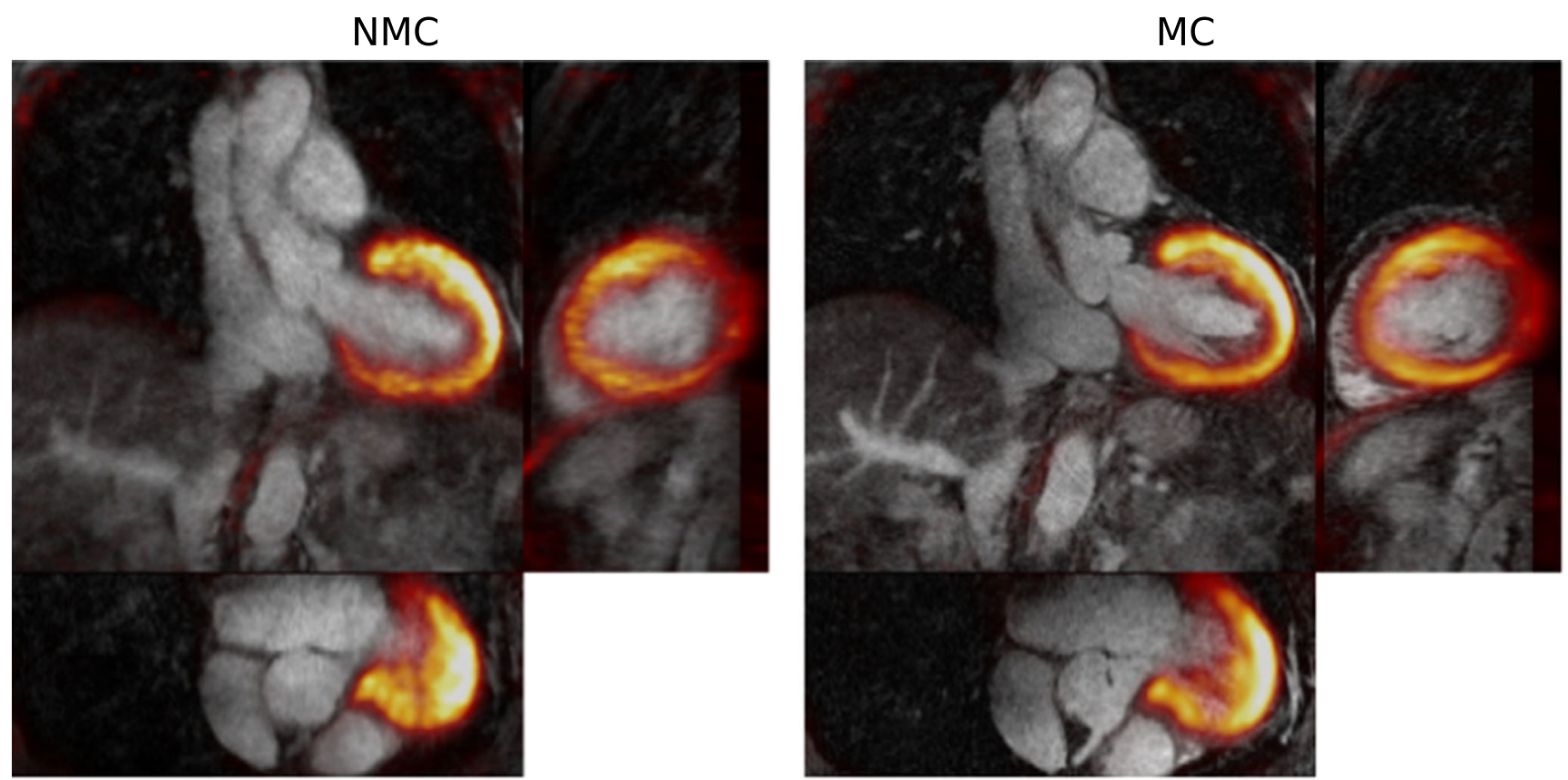

Fig. 9 Example fused PET-CMRA image (Patient 4) showing uncorrected (NMC) and motion-corrected (MC) images. The motion correction framework produces co-registered diagnostic PET and MR images where an improved correspondence between both modalities can be observed 
from appropriate alignment between attenuation maps and emission data in the motion corrected reconstruction framework. This is particularly significant in the anterior wall (Fig. 8a), located closer to the heart-lung interface, which is consistent with findings in recent studies [29]. Our results suggest that respiratory motion compensation is fundamental for avoiding misinterpretation of non-viable segments in the myocardium by ${ }^{18}$ F-FDG PET. An improved correspondence to the anatomy and delineation of transmural and non-transmural myocardial viability defects was observed when comparing LGE-MRI with the motion corrected PET images. Recent studies have compared conventional LGE-MRI and ${ }^{18}$ F-FDG PET for the assessment of myocardial viability [8, 30], finding good agreement between the two modalities. However, both studies report some mismatches between ${ }^{18} \mathrm{~F}$-FDG PET and LGE. It is worth noting that in these studies LGE-MR images were acquired during breath-hold using 2D ECG-triggered sequences, as conventionally done in clinical practice, to minimise cardiac and respiratory motion. On the contrary, in both studies, PET images did not include any motion compensation technique, which could partially explain the mismatches between the two modalities. Further simultaneous ${ }^{18}$ F-FDG PET and LGE-MRI studies with 3D MR acquisition sequences that allow for motion compensation in both modalities (such as the recently proposed BOOST sequence [31]) could allow for a fairer comparison.

This study has a number of limitations. First, the presence of stents in at least one of the coronary arteries for each of the patients limited the number of MR visible vessels available for analysis. A larger cohort would be required to provide a sufficient number of vessels for a more robust statistical analysis of improvements in image quality after motion correction. Second, variations in the overlap between the CMRA and PET acquisitions resulted in high variability in the fraction of PET data that could be motion corrected and therefore in the quality of the PET images. An acquisition protocol with an extended PET list-mode acquisition that guarantees full overlap between the two acquisitions would allow for using the full capabilities of the motion correction approach. Third, patients with known chronic total occlusion were recruited for this study. In order to assess the diagnostic performance of the proposed simultaneous visualisation of myocardial viability and coronary anatomy a similar study would need to be performed in subjects with suspected coronary artery disease.

Our PET-CMRA protocol includes the acquisition of a Dixon-based attenuation correction map, which segments the image into four tissue classes and assigns a fixed attenuation value to each of them [23]. Studies have shown that assigning a fixed attenuation value to the lung tissue can affect accuracy of the PET images in the thoracic region, inducing bias in quantification of lung lesions [32] and volumes of interest within the lungs [33]. However, such effect was shown to be less significant in cardiac structures. Moreover, the study by Lau et al. [34] showed no statistically significant difference in average myocardial uptake when comparing PET-CT and PET-MR using Dixon-based attenuation correction.

The proposed motion compensation technique only addresses the problem of respiratory motion. In CMRA, the problem of cardiac motion was addressed by acquiring data only during the quiescent mid-diastolic period of the cardiac cycle. A similar approach could be used for the PET data by rejecting the fraction of data that falls outside the diastolic period, however, this would impact image quality by increasing the noise in the PET images. Alternatively, if information about cardiac motion is available, it could be included in the PET image reconstruction process [35]. The proposed approach does not include a mechanism for compensating for unpredictable bulk motion of the patient during the PET-CMRA examination. Techniques such as the one proposed by Kolbitsch et al. [36] for detection and correction of whole-body motion in both PET and MR could be adapted to be used with the proposed framework. Finally, in this work, one PET image is independently reconstructed for each respiratory position, and then transformed into the reference end-expiratory position. This approach has been shown to introduce bias due to the reduced number of counts in each respiratory position in simulation studies [37, 38]. Future work will include the deformation fields directly in the motion compensated PET reconstruction process by modifying the system matrix [14].

\section{Conclusion}

We have presented a first clinical validation of a novel respiratory motion-corrected whole-heart PET-CMRA framework for simultaneous visualisation of coronary anatomy and myocardial integrity in patients with coronary artery disease. The framework allows for acquiring diagnostic images with both modalities in a time efficient examination. The reported results have shown that motion correction improves image quality for both modalities compared to the uncorrected images. A good agreement between coronary anatomy depicted by motion-corrected CMRA and X-ray angiography was observed. In addition, motion-corrected ${ }^{18}$ F-FDG PET images were in good agreement with LGE-MRI, showing more accurate depiction of both transmural and non-transmural viability defects.

Acknowledgements This work was supported by the King's College London \& Imperial College London EPSRC Centre for Doctoral Training in Medical Imaging (EP/L015226/1), the German Research Council (DFG/8810001759), the Wellcome EPSRC Centre for Medical Engineering (WT 203148/Z/16/Z) and the following grants: (1) EPSRC EP/N009258/1, (2) MRC MR/L009676/1. The research was supported by the National Institute for Health Research (NIHR) Biomedical Research Centre based at Guy's and St Thomas' NHS Foundation Trust and King's College London. The views expressed are those of the authors and not necessarily those of the NHS, the NIHR or the Department of Health. 


\section{Compliance with ethical standards}

Conflict of interest The authors declare that they have no conflict of interest.

Ethical approval All procedures performed in studies involving human participants were in accordance with the ethical standards of the institutional ethics committee and with the 1964 Helsinki declaration and its later amendments or comparable ethical standards.

Informed consent Written informed consent was obtained from all individual participants included in the study.

Open Access This article is distributed under the terms of the Creative Commons Attribution 4.0 International License (http:// creativecommons.org/licenses/by/4.0/), which permits unrestricted use, distribution, and reproduction in any medium, provided you give appropriate credit to the original author(s) and the source, provide a link to the Creative Commons license, and indicate if changes were made.

\section{References}

1. Rischpler C, Nekolla SG, Dregely I, Schwaiger M. Hybrid PET/ MR imaging of the heart: potential, initial experiences, and future prospects. J Nucl Med. 2013;54:402-15.

2. Ratib O, Nkoulou R. Potential applications of PET/MR imaging in cardiology. J Nucl Med. 2014;55:40S-6S.

3. Rischpler C, Nekolla SG, Kunze KP, Schwaiger M. PET/MRI of the heart. Semin Nucl Med. 2015;45:234- 47.

4. Abgral R, Dweck MR, Trivieri MG, Robson PM, Karakatsanis N, Mani V, et al. Clinical utility of combined FDG-PET/MR to assess myocardial disease. J Am Coll Cardiol Img. 2017;10:594-7.

5. Schwaiger M, Kunze K, Rischpler C, Nekolla SG. PET/MR: yet another Tesla? J Nucl Cardiol. 2017;24:1019-31.

6. Dweck MR, Abgral R, Trivieri MG, Robson PM, Karakatsanis N, Mani V, et al. Hybrid magnetic resonance imaging and positron emission tomography with fluorodeoxyglucose to diagnose active cardiac sarcoidosis. J Am Coll Cardiol Img. 2017;11:94-107.

7. Nensa F, Kloth J, Tezgah E, Poeppel TD, Heusch P, Goebel J, et al. Feasibility of FDG-PET in myocarditis: Comparison to CMR using integrated PET/MRI. J Nucl Cardiol. 2016. https://doi.org/10.1007/ s12350-016-0616-y.

8. Rischpler C, Langwieser N, Souvatzoglou M, Batrice A, van Marwick S, Snajberk J, et al. PET/MRI early after myocardial infarction: evaluation of viability with late gadolinium enhancement transmurality vs. $18 \mathrm{~F}-\mathrm{FDG}$ uptake. Eur Hear J Cardiovasc Imaging. 2015;16:661-9.

9. Robson PM, Dweck MR, Trivieri MG, Abgral R, Karakatsanis NA, Contreras J, et al. Coronary artery PET/MR imaging: feasibility, limitations, and solutions. J Am Coll Cardiol Img. 2017;10:1103-12.

10. Henningsson M, Botnar RM. Advanced respiratory motion compensation for coronary MR angiography. Sensors. 2013;13:6882-99.

11. Rahmim A, Rousset O, Zaidi H. Strategies for motion tracking and correction in PET. PET Clin. 2007;2:251-66.

12. Munoz C, Kolbitsch C, Reader AJ, Marsden P, Schaeffter T, Prieto C. MR-based cardiac and respiratory motion-compensation techniques for PET-MR imaging. PET Clin. 2016;11:179-91.

13. Picard Y, Thompson CJ. Motion correction of PET images using multiple acquisition frames. IEEE Trans Med Imaging. 1997;16:137-44.
14. Qiao F, Pan T, Clark JW, Mawlawi OR. A motion-incorporated reconstruction method for gated PET studies. Phys Med Biol. 2006;51:3769-83.

15. Manber R, Thielemans K, Hutton B, Barnes A, Ourselin S, Arridge $\mathrm{S}$, et al. Practical PET respiratory motion correction in clinical PET/ MR. J Nucl Med. 2015;56:890-6.

16. Küstner T, Schwartz M, Martirosian P, Gatidis S, Seith F, Gilliam C, et al. MR-based respiratory and cardiac motion correction for PET imaging. Med Image Anal. 2017;42:129-44.

17. Kolbitsch C, Ahlman MA, Davies-Venn C, Evers R, Hansen M, Peressutti D, et al. Cardiac and respiratory motion correction for simultaneous cardiac PET/MR. J Nucl Med. 2017;58:846-52.

18. Munoz C, Neji R, Cruz G, Mallia A, Jeljeli S, Reader AJ, et al. Motion-corrected simultaneous cardiac positron emission tomography and coronary MR angiography with high acquisition efficiency. Magn Reson Med. 2017;79:339-50.

19. Henningsson M, Koken P, Stehning C, Razavi R, Prieto C, Botnar RM. Whole-heart coronary MR angiography with $2 \mathrm{D}$ self-navigated image reconstruction. Magn Reson Med. 2012;67:437-45.

20. Cruz G, Atkinson D, Henningsson M, Botnar RM, Prieto C. Highly efficient nonrigid motion-corrected $3 \mathrm{D}$ whole-heart coronary vessel wall imaging. Magn Reson Med. 2017;77:1894-908.

21. Prieto C, Doneva M, Usman M, Henningsson M, Greil G, Schaeffter T, et al. Highly efficient respiratory motion compensated free-breathing coronary MRA using golden-step Cartesian acquisition. J Magn Reson Imaging. 2015;41:738-46.

22. Botnar RM, Stuber M, Danias PG, Kissinger KV, Manning WJ. Improved coronary artery definition with T2-weighted, free-breathing, three-dimensional coronary MRA. Circulation. 1999;99:3139-48.

23. Martinez-Möller A, Souvatzoglou M, Delso G, Bundschuh RA, Chefd'hotel C, Ziegler SI, et al. Tissue classification as a potential approach for attenuation correction in whole-body PET/MRI: evaluation with PET/CT data. J Nucl Med. 2009;50:520-6.

24. Nuyts J, Bal G, Kehren F, Fenchel M, Michel C, Watson C. Completion of a truncated attenuation image from the attenuated PET emission data. IEEE Trans Med Imaging. 2013;32:237-46.

25. Batchelor PG, Atkinson D, Irarrazaval P, Hill DLG, Hajnal J, Larkman D. Matrix description of general motion correction applied to multishot images. Magn Reson Med. 2005;54:1273-80.

26. Etienne A, Botnar RM, Van Muiswinkel AMC, Boesiger P, Manning WJ, Stuber M. "Soap-bubble" visualization and quantitative analysis of 3D coronary magnetic resonance angiograms. Magn Reson Med. 2002;48:658-66.

27. Cerqueira MD, Weissman NJ, Dilsizian V, Jacobs AK, Kaul S, Laskey WK, et al. Standardized myocardial segmentation and nomenclature for tomographic imaging of the heart. J Cardiovasc Magn Reson. 2002;4:203-10.

28. Nekolla SG, Miethaner C, Nguyen N, Ziegler SI, Schwaiger M. Reproducibility of polar map generation and assessment of defect severity and extent assessment in myocardial perfusion imaging using positron emission tomography. Eur J Nucl Med. 1998;25: 1313-21.

29. Lassen ML, Rasul S, Beitzke D, Stelzmüller M-E, Cal-Gonzalez J, Hacker M, et al. Assessment of attenuation correction for myocardial PET imaging using combined PET/MRI. J Nucl Cardiol. 2017. https://oi.org/10.1007/s12350-017-1118-2.

30. Bulluck H, White SK, Fröhlich GM, Casson SG, O'Meara C, Newton A, et al. Quantifying the area at risk in Reperfused STsegment-elevation myocardial infarction patients using hybrid cardiac positron emission tomography-magnetic resonance imaging. Circ Cardiovasc Imaging. 2016;9:e39.

31. Ginami G, Neji R, Rashid I, Chiribiri A, Ismail TF, Botnar RM, et al. $3 \mathrm{D}$ whole-heart phase sensitive inversion recovery CMR for simultaneous black-blood late gadolinium enhancement and bright-blood coronary CMR angiography. J Cardiovasc Magn Reson. 2017;19:94. 
32. Rauscher I, Eiber M, Furst S, Souvatzoglou M, Nekolla SG, Ziegler SI, et al. PET/MR imaging in the detection and characterization of pulmonary lesions: technical and diagnostic evaluation in comparison to PET/CT. J Nucl Med. 2014;55:724-9.

33. Marshall HR, Prato FS, Deans L, Theberge J, Thompson RT, Stodilka RZ. Variable lung density consideration in attenuation correction of whole-body PET/MRI. J Nucl Med. 2012;53:977-84.

34. Lau JMC, Laforest R, Sotoudeh H, Nie X, Sharma S, McConathy J, et al. Evaluation of attenuation correction in cardiac PET using PET/MR. J Nucl Cardiol. 2017;24:839-46.

35. Munoz C, Neji R, Botnar RM, Prieto C. Highly efficient respiratory motion corrected dual-phase coronary MR angiography in a 3T
PET-MR system. In Proceedings of the 25th Annual Meeting of ISMRM, Honolulu. 2017. p 1025.

36. Kolbitsch C, Prieto C, Tsoumpas C, Schaeffter T. A 3D MRacquisition scheme for nonrigid bulk motion correction in simultaneous PET-MR. Med Phys. 2014;41:82304.

37. Dikaios N, Izquierdo-Garcia D, Graves MJ, Mani V, Fayad ZA, Fryer TD. MRI-based motion correction of thoracic PET: initial comparison of acquisition protocols and correction strategies suitable for simultaneous PET/MRI systems. Eur Radiol. 2012;22:439-46.

38. Polycarpou I, Tsoumpas C, Marsden PK. Analysis and comparison of two methods for motion correction in PET imaging. Med Phys. 2012;39:6474-83. 